\title{
Reporte de un caso de válvula aórtica unicúspide: un diagnóstico ecocardiográfico
}

\author{
Eric Bogantes Pereira* \\ Ulises López Cardoza* \\ Kirsten Alvarado Rodríguez**
}

* Médico Asistente Especialista en Cardiología. Hospital México. Caja Costarricense del Seguro Social. San José. Costa Rica

** Médico Residente de Cardiología. Universidad de Costa Rica. Caja Costarricense del Seguro Social. San José. Costa Rica

Recibido:01/04/2020

Aceptado: 18/10/2020

En línea: 31/12/2020

Citar como: Bogantes Pereira E, López Cardoza U, Alvarado Rodríguez K. Reporte de un caso de válvula aórtica unicúspide: un diagnóstico ecocardiográfico. Rev Ecocar Pract (RETIC). 2020 (Dic); 3 (3): 22-25. doi: 10.37615/retic.v3n3a10.

Cite this as: Bogantes Pereira E, López Cardoza U, Alvarado Rodríguez K. Report of a case of unicuspid aortic valve: an echocardiographic diagnosis. Rev Ecocar Pract (RETIC). 2020 (Dic); 3 (3): 22-25.doi: 10.37615/retic.v3n3a10

\section{Palabras clave \\ $\triangleright$ Válvula aórtica unicúspide \\ $\triangleright$ Ecocardiografía \\ transesofágica \\ $\triangleright$ Estenosis aórtica}

\begin{tabular}{l}
\hline Keywords \\
\hline$\triangleright$ Unicuspid aortic valve \\
$\triangleright$ Transesophageal \\
echocardiography \\
$\triangleright$ Aortic stenosis \\
\end{tabular}

\section{Presentación del caso}

Varón de 56 años, sin antecedentes patológicos previos. Presentó una historia de un mes de evolución de disnea de pequeños esfuerzos, edema de miembros inferiores y ortopnea. Finalmente, ingresó en Urgencias con datos de insuficiencia cardíaca descompensada y un soplo sistólico grado IVNI en foco aórtico. Se le realizó ecocardiografía transtorácica (ETT) que documentó dilatación ventricular izquierda con fracción de eyección del ventrículo izquierdo (FEVI) reducida y disfunción diastólica. Además, llamaba la atención una estenosis aórtica moderada que tenía asociado un jet de insuficiencia que impresionaba ser excéntrico, con dilatación de raíz de aorta y aorta ascendente, sin poder esclarecer la morfología valvular aórtica.

Se valoró de forma más detallada la válvula aórtica con ecocardiografía transesofágica (ETE), donde se apreciaba una válvula aórtica unicúspide de configuración unicomisural con un jet de insuficiencia aórtica excéntrica severa asociada a estenosis aórtica moderada (velocidad pico $3.27 \mathrm{~m} / \mathrm{s}$; gradiente pico $43 \mathrm{mmHg}$; gradiente medio $23 \mathrm{mmHg}$; área valvular aórtica $1.56 \mathrm{~cm}^{2}$ ) (Figura 1, Vídeo 1 a Vídeo 3) y dilatación aórtica con diámetro máximo de 54 mm (Figura 2 y Vídeo 4).

Se realizó angiotomografía computarizada (angio-TC) que documentó dilatación aneurismática de la raíz y arco aórticos de hasta $54 \mathrm{~mm}$; el cateterismo reportó coronarias sin lesiones angiográficamente significativas, y en el aortograma por angiografía se midió la aorta ascendente de $55 \mathrm{~mm}$. El paciente fue estabilizado de su insuficiencia cardíaca. Se discutió el caso de forma multidisciplinaria y con el paciente, y se plantearon diferentes posibilidades dada la falla ventricular y la dilatación aórtica. Sin embargo, el paciente decidió no realizarse ninguna intervención. Por tanto, se continuó su manejo médico para la insuficiencia cardíaca.

\section{Estudio por imagen}

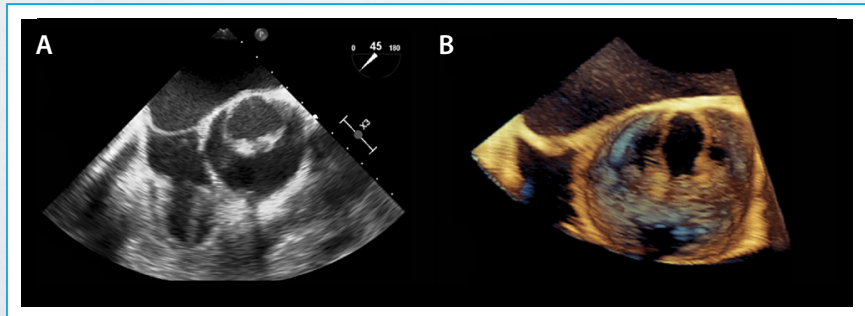

Figura 1. ETE a $45^{\circ}$ que muestra la morfología típica de la válvula aórtica unicúspide (A: imagen 2D; B: imagen 3D) 


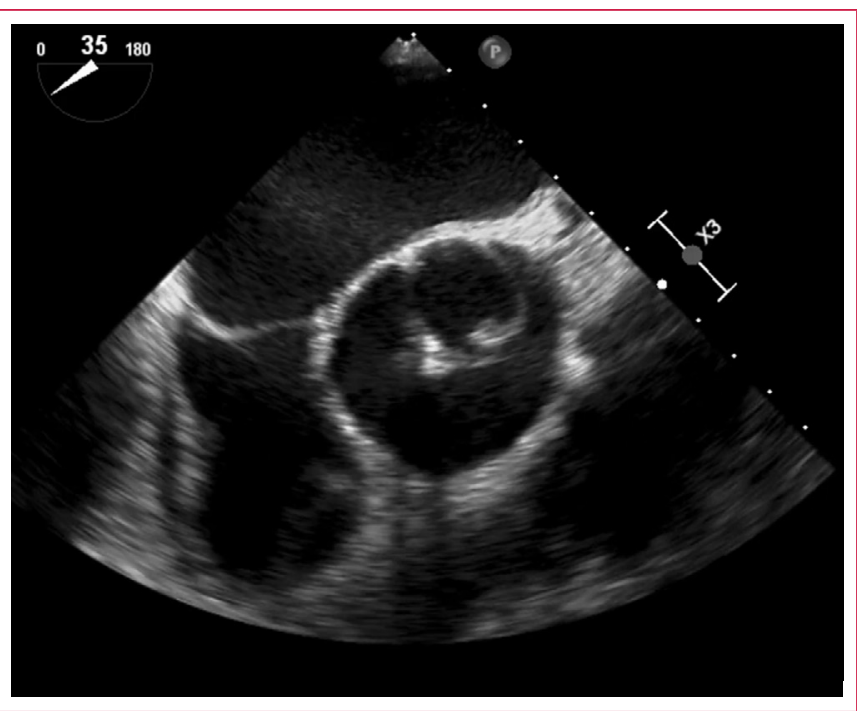

Vídeo 1. ETE a $45^{\circ}$ en modo 2D. Apertura y cierre de la válvula aórtica unicúspide

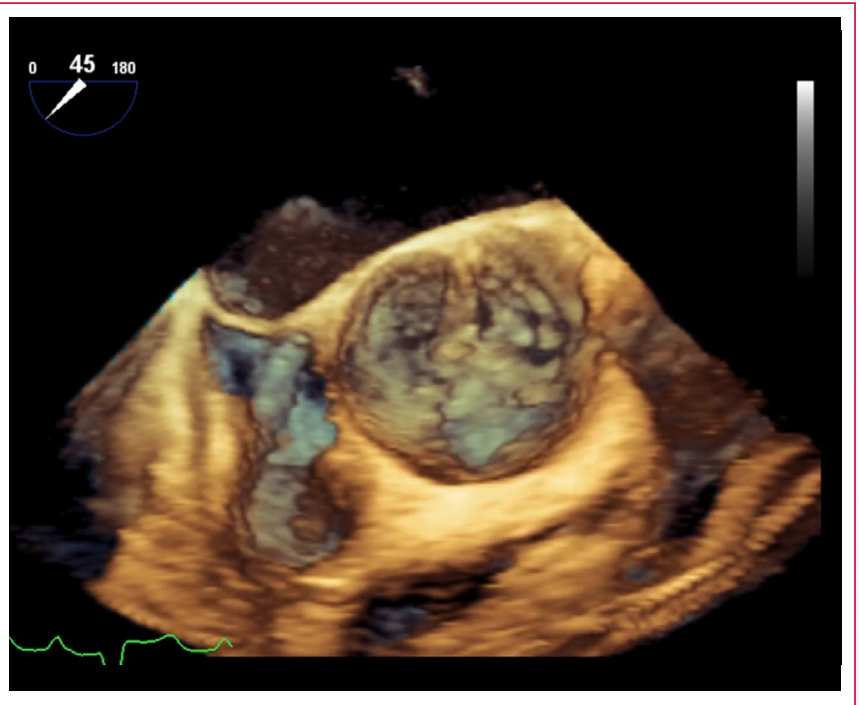

Vídeo 2. ETE a $45^{\circ}$ en modo 3D. Reconstrucción de la válvula aórtica

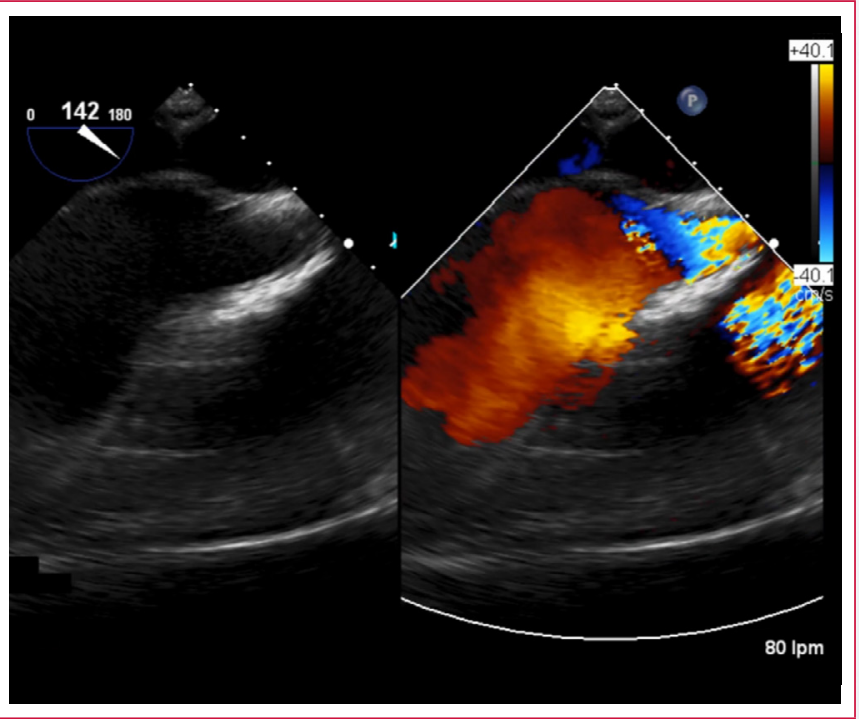

Vídeo 3. ETE a $140^{\circ}$ en modo Doppler color. Se pueden ver los jets de estenosis e insuficiencia aórtica

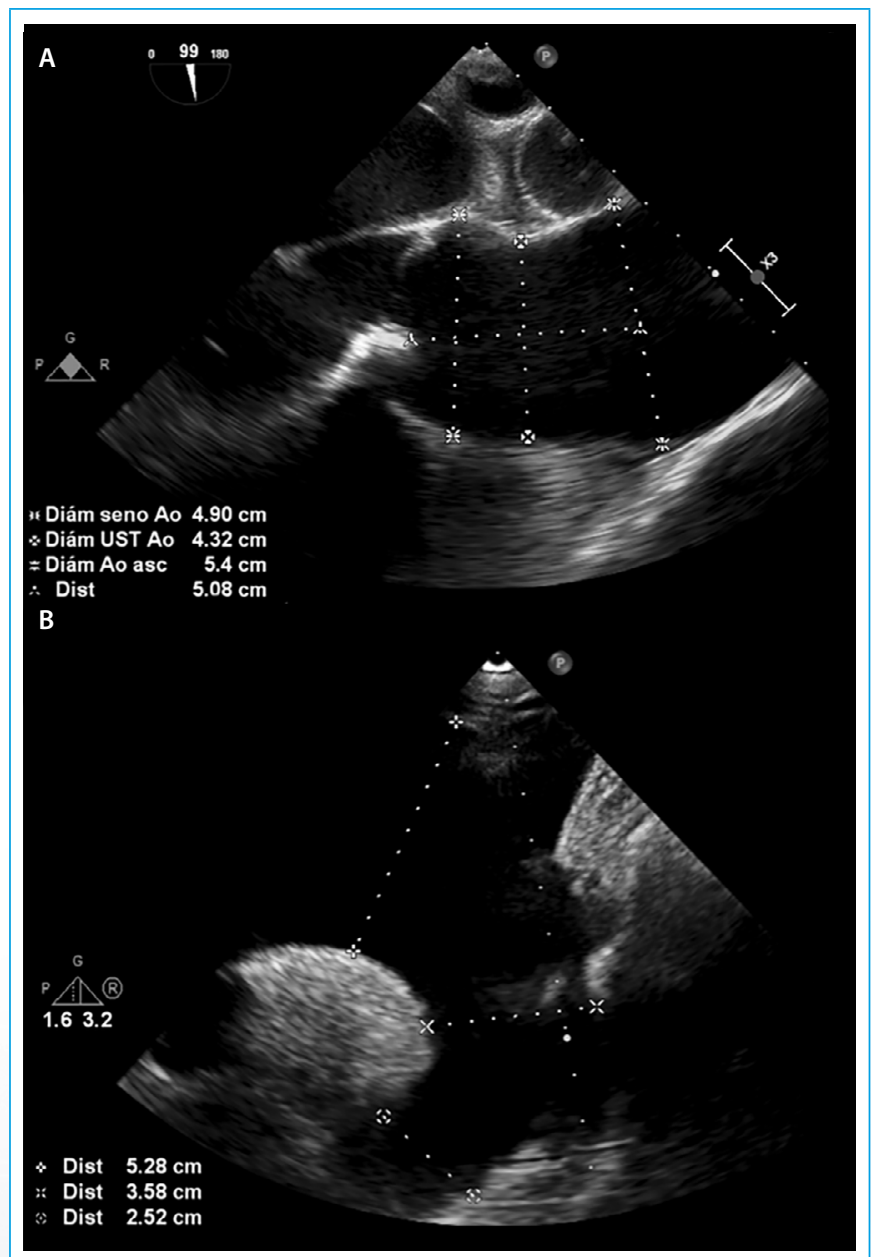

Figura 2. ETE (A) y ETT (B) de las posibles morfologías de la válvula aórtica unicúspide que muestran la dilatación de la aorta ascendente, una complicación frecuentemente asociada a la válvula aórtica bicúspide

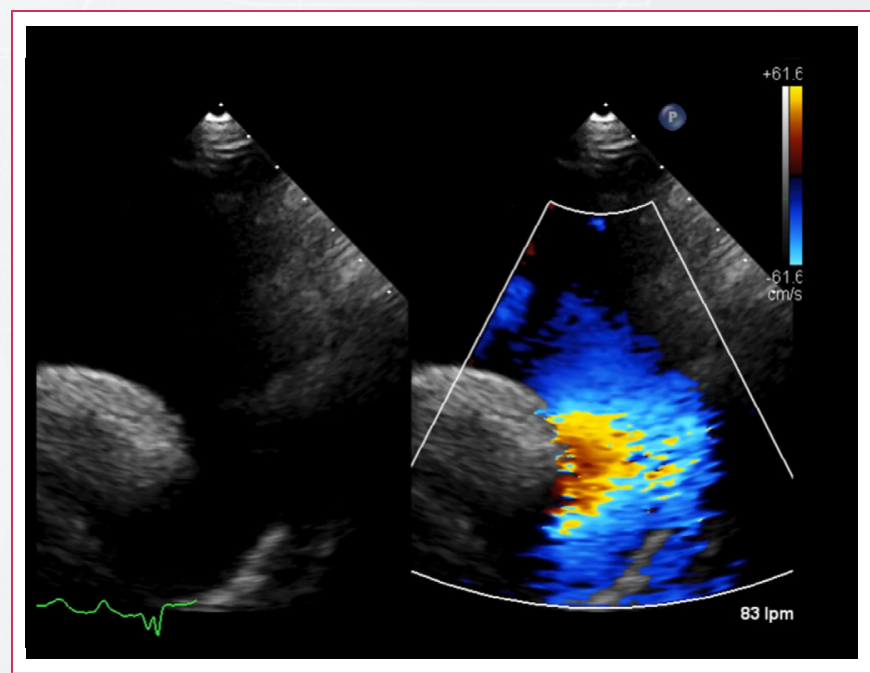

Vídeo 4. ETT supraesternal eje largo, arco dilatado, flujo retrógrado iastólico de insuficiencia aortica

\section{Discusión}

La válvula aórtica unicúspide es una patología congénita rara, con una incidencia muy baja que supone un $0.02 \%$ en pacientes adultos y menos del $5 \%$ 
de los pacientes para reemplazo valvular aórtico ${ }^{(1)}$. Predomina en hombres, a razón 4:1.

La malformación de las valvas causa mayor calcificación y fibrosis, usualmente entre los 30-50 años, llevando a una estenosis progresiva, junto con regurgitación valvular. El diagnóstico era previamente realizado en la autopsia o con la inspección intraoperatoria de la válvula, sin embargo, el avance de las técnicas de imagen ecocardiográfica ha permitido una evaluación no invasiva más temprana(2).

Su etiología se remonta a la embriogénesis, ya que las tres valvas con sus comisuras se desarrollan de los tubérculos embrionarios del tronco aórtico. En el caso de la válvula unicúspide, hay un fallo en el desarrollo de dos comisuras, lo que genera un par de rafes donde deberían ubicarse normalmente estas comisuras ${ }^{(1)}$. Es importante destacar que los senos de Valsalva también se originan de estos tubérculos embrionarios, pero su desarrollo concluye usualmente de forma normal. Por ello, estos pacientes generalmente tienen arterias coronarias en posición normal ${ }^{(1)}$.

Esta morfología puede clasificarse en dos tipos, válvula unicúspide unicomisural y acomisural, según exista o no unión lateral a la aorta a nivel del orificio de apertura (Figura 3). En las unicomisurales (Figura 3B), hay una unión lateral a la aorta y la única cúspide se encuentra alrededor del orificio aórtico; esta configuración, por lo general, tiene un área de orificio efectivo mayor que su contraparte. Las acomisurales (Figura 3A) tienen una apertura más triangular causada por un pobre desarrollo de las tres cúspides ${ }^{(3)}$.
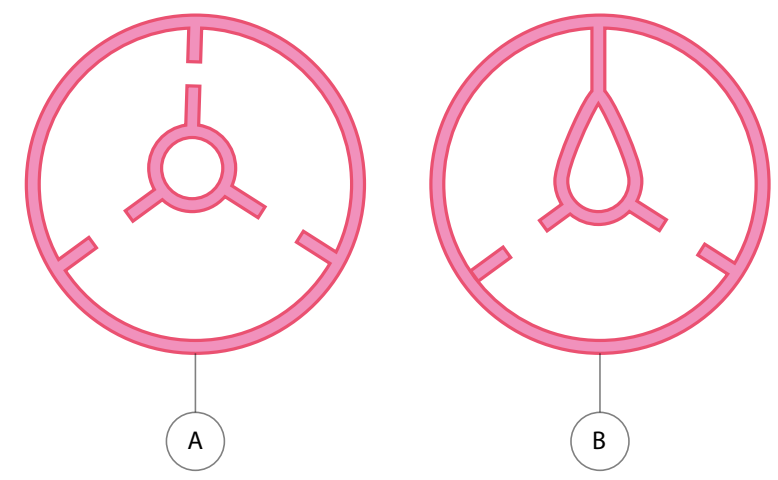

Figura 3. Diagrama de las posibles morfologías de la válvula aórtica unicúspide: acomisural (A) y unicomisural (B)

Clínicamente, predomina la estenosis aórtica en un 92\% de los casos, con o sin regurgitación aórtica. En estos casos, la calcificación aórtica llega a ser severa, y es importante tenerlo en cuenta cuando esta calcificación se extiende hacia el septum interventricular, lo cual puede resultar en daños al tejido de conducción durante el desbridamiento para el reemplazo valvular ${ }^{(4)}$ así como trastornos de la conducción. Los síntomas tienden a presentarse 20-30 años antes que en los pacientes con válvulas tricúspides. Además, tiene una presentación bimodal, dado que se comporta de forma menos agresiva en pacientes mayores y más agresiva en pacientes jóvenes ${ }^{(5)}$.

Su diagnóstico se basa en la imagen cardíaca. Las imágenes 3D de la ETE son un pilar para el reconocimiento de la morfología de esta valvulopatía. El reto principal está en que, durante la diástole, el rafe usualmente impresiona una comisura verdadera en el eje corto. Sin embargo, en sístole no existe una separación de cúspides, sino que tiene una apertura valvular excéntrica, en forma de "gota de agua"(1). El diagnóstico correcto puede complicarse por la calcificación y el número de comisuras a nivel de la unión sinotubular ${ }^{(2)}$.
Es necesario valorar también la raíz y aorta ascendente puesto que se ha demostrado hasta un 50\% de casos de válvulas unicúspides con una aorta ascendente de más de 40 mm y que necesitan reemplazo aórtico(1) ${ }^{(1)}$ como el caso de nuestro paciente. También las válvulas bicúspides y unicúspides tienen mayor riesgo de disección ${ }^{(3)}$.

Existe un registro europeo que plantea ciertos criterios ecocardiográficos para distinguir la morfología unicúspide ${ }^{(2)}$. Dentro de los criterios mayores figuran: zona de unión comisural única, forma redondeada en la zona opuesta a la unión comisural, orificio valvular excéntrico durante la sístole y edad menor de 20 años con un gradiente transvalvular medio mayor de $15 \mathrm{mmHg}$. Por otra parte, la aortopatía torácica asociada y la edad menor de 40 años fueron definidos como criterios menores. En este registro, la presencia de 3 criterios mayores o 2 criterios mayores con 1 criterio menor se encontró en todos los pacientes con válvulas unicúspides y en ninguno de los pacientes con morfología bicúspide o tricúspide, con una buena sensibilidad y especificidad ${ }^{(2)}$.

El tratamiento se basa en el reemplazo valvular aórtico, con o sin sustitución aórtica según el tamaño del vaso(6). La intervención valvular y de la aorta juntas no conlleva mayor mortalidad, sino, más bien, la intervención conjunta tiene mejores resultados a largo plazo que solamente cirugía valvular ${ }^{(7)}$.

La cirugía en válvulas bicúspides con estenosis tiene mejoría significativa en la FEVI, con respecto a los pacientes con regurgitación, los cuales tienen mayor índice de eventos cardiovasculares y tienden a continuar con disfunción ventricular ${ }^{(8)}$. De hecho, en estos pacientes se documentó un punto de corte para el diámetro diastólico del ventrículo izquierdo (DDVI) de $60 \mathrm{~mm}$ como predictor independiente para la no mejoría de la FEVI posterior a la cirugía. En ellos también se recomienda una intervención temprana antes de que disminuya la FEVI y aumente el DDVI(8).

\section{Conclusión}

La ETE y las nuevas técnicas de imagen 3D son clave para el diagnóstico temprano de las lesiones de la válvula aórtica. La descripción morfológica es indispensable para el planeamiento quirúrgico de estos pacientes, así como la búsqueda de otras anomalías asociadas como patologías de aorta y determinar la presencia de disfunción ventricular izquierda para realizar una intervención temprana y evitar el daño irreversible del ventrículo izquierdo.

\section{Ideas para recordar}

- El avance de las técnicas de imagen ecocardiográfica transtorácica y transesofágica han permitido una mejor y más temprana evaluación de anomalías valvulares como la aórtica unicúspide, para lograr una intervención más temprana en estos casos.

- Es importante no limitar la valoración ecocardiográfica a la valvulopatía aórtica, sino también incluir la valoración de la raíz aórtica y aorta ascendente dentro de la evaluación de pacientes con sospecha de esta patología.

- Existe una serie de criterios mayores y menores que permiten distinguir con buena especificidad y sensibilidad una válvula bicúspide de una unicúspide, cuando existen dudas de la morfología valvular aórtica.

\section{Bibliografía}

1. Sniecinski, R, Shanewise J, Glas, K. Transesophageal echocardiography of a unicuspid aortic valve. Anesthesia \& Analgesia 2009; 108 (3): 788-789. 
2. Ewen S, Karliova I, Weber P, Schirmer S, Abdul-Khaliq H, Schöpe J, Mahfoud F Schäfers H. Echocardiographic criteria to detect unicuspid aortic valve morphology. European Heart Journal-Cardiovascular Imaging 2019; 20 (1): 40-44.

3. Singh D, Chee T. Incidental diagnosis of unicuspid aortic valve in an asymptomatic adult. Journal of the American Society of Echocardiography 2008; 21 (7): 876.e5

4. Mookadam F, Thota V, García A, Mohsen E, Zamorano J, Khandheria B. Unicuspid aortic valve in adults. A systematic review. J Heart Valve Dis 2010; 19 (1).

5. Singh S, Ghayal P, Mathur A, Mysliwiec M, Lovoulos C, Solanki P, Klapholz M, Maher J. Unicuspid unicommissural aortic valve. An extremely rare congenital anomaly. Texas Heart Institute Journal 2015; 42 (3): 273-276.
6. Ingason A, Sigfusson G, Torfason B. Congenital aortic stenosis due to unicuspid unicommissural aortic valve: a case report. Journal of Cardiothoracic Surgery 2018; 13 (1).

7. Zhu Y, Roselli E, Idrees J, Wojnarski C, Griffin B, Kalahasti V, Pettersson G, Svensson L. Outcomes after operations for unicuspid aortic valve with or without ascending repair in adults. The Annals of Thoracic Surgery 2016; 101 (2): 613-619.

8. Disha K, Espinoza A, Rouman M, Secknus M, Kuntze T, Girdauskas E. Longterm recovery of reduced left ventricular ejection fraction after aortic valve replacement in patients with bicuspid aortic valve disease. Thorac Cardiovasc Surg 2016 Aug; 64 (5): 418-426. 